\title{
Obtención de circonas estabilizadas (Ca,Mg-PSZ) nanocristalinas a partir de mezclas de dolomita y circona monoclínica mediante molienda de alta energía
}

\author{
J. CARRETERO, M. A. SAINZ, S. SERENA, A. CABALLERO \\ Instituto de Cerámica y Vidrio, (C.S.I.C.), Cantoblanco, Madrid, Spain.
}

\begin{abstract}
El objetivo del presente trabajo es la obtención de materiales cerámicos de circona estabilizada (Ca,Mg-PSZ) nanocristalina mediante molienda de alta energía, a partir de circona monoclínica comercial y una materia prima de alta pureza y bajo coste como es la dolomita $\left(\mathrm{CaMg}\left(\mathrm{CO}_{3}\right)_{2}\right)$. La molienda de alta energía de mezclas, especialmente en la que al menos uno de los componentes contenga un grupo carbonato, hidroxilo o se lleve a cabo en presencia de agua, se ha convertido en un método alternativo para obtener materiales nanocristalinos.

En este trabajo, mediante un molino de bolas de alta energía se han obtenido nanopartículas de circona monoclínica y dolomita con un tamaño medio de cristalito de $20 \pm 5 \mathrm{~nm}$. A partir de estas se han preparado materiales cerámicos densos $(98 \%$ $\rho / \rho_{\mathrm{t}}$ ) de circona (Ca, Mg-PSZ) nanocristalina por sinterización a $1200^{\circ}$ y $1300^{\circ} \mathrm{C}$ durante 18 y 48 minutos.
\end{abstract}

Palabras clave: circona, dolomita, nanocristalino, molienda de alta energía, sinterización.

Stabilized zirconia (Ca, Mg-PSZ) nanocrystalline from dolomite and monoclinic zirconia by means of high-energy milling.

The objective of the present work is to obtain ceramic materials of stabilized zirconia (Ca,Mg-PSZ) nanocrystalline by means of high-energy milling, starting from monoclinic zirconia and a raw material of high purity and low cost like the dolomite $\left(\mathrm{CaMg}\left(\mathrm{CO}_{3}\right)_{2}\right)$. The high-energy milling of mixtures, especially in those that at least one of the components contains a carbonate or hidroxyl group or it is carried out in presence of water, it has become an alternative method to obtain nanocrystalline materials.

In this work using high-energy ball-milling, nanopartícles of monoclinic zirconia and dolomite have been obtained with a average size of crystallite about $20 \pm 5 \mathrm{~nm}$. Starting from these, dense stabilized zirconia (Ca,Mg-PSZ) nanocrystalline materials have been obtained $\left(98 \% \rho / \rho_{\mathrm{t}}\right)$ for sintering at $1200^{\circ}-1300^{\circ} \mathrm{C}$ during $18-48$ minutes.

Key words: zirconia, dolomite, nanocrystalline, high-energy milling, sintering.

\section{INTRODUCCIÓN}

Los cambios producidos en la última década en Ciencia y Tecnología de Materiales, y en especial en el diseño y producción de materiales cerámicos avanzados, han estado ligados a la utilización de materias primas naturales o de síntesis con granulométrias ultrafinas, del orden del nanómetro $\left(10^{-9} \mathrm{~m}\right), \mathrm{y}$ más íntima y homogéneamente mezcladas, para la obtención de materiales cerámicos "nanocristalinos"(1). En la actualidad existe un interés creciente en el estudio y procesamiento de este tipo de materiales, diseñados intencionadamente con una o más dimensiones por debajo de $100 \mathrm{~nm}$, debido a la observación en ellos de unas propiedades nuevas y/o superiores a las existentes a escala micrométrica (conductividad térmica extremadamente baja, alta refractariedad, aumento de la resistencia a la fractura por fatiga, alta dureza, superplasticidad, etc.)(2,3).

Estos materiales cerámicos nanocristalinos pueden presentar microestructuras con precipitados ultrafinos dispersos en una matriz o en los límites de grano (nanocompuestos), fases simples enteramente compuestas de granos ultrafinos o conteniendo más de una fase en la que los granos de todas las fases presentes sean menores de $100 \mathrm{~nm}$ (4).

La obtención de polvos nanoparticulados puede ser lle- vada a cabo por: síntesis química por precipitación de una solución, CVD, etc.; síntesis termo-física dominada por la técnica de condensación de fase vapor, y síntesis mecánica por el método Mecano-Químico mediante molienda de alta energía(5-8). En esta última síntesis, tras el tratamiento mecánico de los polvos de uno o más componentes, se han observado fenómenos asociados a la "activación mecánica" que incluyen: la reducción del tamaño de partícula/cristalito y consecuentemente un aumento de la superficie específica, la amorfización de las fases cristalinas, creación de defectos estructurales, transformaciones de fase, transiciones orden-desorden, cristalización a partir de estados amorfos y reacciones químicas que han permitido el desarrollo de fases metaestables con propiedades físicas y químicas únicas. Las ventajas de la molienda de alta energía frente a reacciones de estado sólido convencionales y procesos químicos en disolución, se hallan en su capacidad para preparar directamente polvos nanoparticulados en grandes cantidades a temperatura ambiente a partir de materias primas de bajo costo, la utilización de equipos relativamente baratos y su simplicidad. En la actualidad este método es empleado en la obtención de diversos tipos de ma- 
teriales cerámicos avanzados(9-12) y resulta particularmente eficaz en el procesamiento de polvos en presencia de agua o en los que al menos uno de los componentes sea un hidróxilo, óxido, carbonato, nitruro, carburo, geles de óxidos hidratados e incluso complejos inorgánicos-orgánicos, debido al efecto activador que tiene sobre la superficie de las partículas(13-15).

En el caso de los materiales de $\mathrm{ZrO}_{2}$, la obtención y consolidación de polvos cerámicos nanoparticulados de $\mathrm{ZrO}_{2}$ ha permitido fabricar materiales con microestructuras extremadamente homogéneas y densidades prácticamente teóricas(16), que presentan mejores propiedades a alta temperatura(17) que los micrométricos.

Entre las numerosas técnicas y métodos que permiten obtener polvos nanopartículados de $\mathrm{ZrO}_{2}$, caben destacar las siguientes: síntesis de plasma por microondas(18), co-precipitación a partir de una solución(19), pirolisis(20), condensación de gas inerte(21) y sol-gel(22). No obstante, los procesos enumerados presentan parámetros de procesado muy críticos y son relativamente costosos por lo que se buscan alternativas más simples y económicas.

En el presente trabajo se propone utilizar un proceso de activación mecánica de los materiales de partida, mediante molienda de alta energía, para la obtención de circona estabilizada (Ca,Mg-PSZ) nanocristalina a partir de circona monoclínica comercial y de una materia prima natural de elevada pureza y bajo coste como es la dolomita. Esta última aportará simultáneamente $\mathrm{CaO}$ y $\mathrm{MgO}$, conocidos óxidos estabilizadores de la $\mathrm{ZrO}_{2}$ (23-25). La activación mecánica ejercida durante la molienda de alta energía, reduce el tamaño de partícula e introduce una alta concentración de defectos en el material (dislocaciones, límites de grano de alto ángulo, etc.)(5). Se consigue de esta manera activar el proceso de sinterización y consecuentemente reducir la temperatura y el tiempo necesario para densificar el material(26). La disminución de la temperatura de tratamiento proporciona un mayor control sobre el crecimiento de grano en el material densificado(27).

\section{MATERIALES}

Se ha empleado una circona $\left(\mathrm{ZrO}_{2}\right)$ comercial de la marca Tosoh Co. (Japón) en forma de polvo atomizado, con estructura monoclínica. El análisis químico de las impurezas mayoritarias fue (\% peso): $0.005 \mathrm{Al}_{2} \mathrm{O}_{3^{\prime}} 0.006 \mathrm{SiO}_{2^{\prime}} 0.003 \mathrm{Fe}_{2} \mathrm{O}_{3}$ y 0.016 $\mathrm{Na}_{2} \mathrm{O}$.

La dolomita $\left(\mathrm{CaMg}\left(\mathrm{CO}_{3}\right)_{2}\right)$ seleccionada fue suministrada por la empresa PRODOMASA (Coín, Málaga), en forma de polvo micronizado de elevada pureza. Su composición química fue (\% peso): $47.70 \mathrm{CO}_{2}, 30.29 \mathrm{CaO}, 22.05 \mathrm{MgO}, 0.018 \mathrm{SiO}_{2^{\prime}}$ $0.011 \mathrm{Al}_{2} \mathrm{O}_{3}$ y $0.008 \mathrm{Fe}_{2} \mathrm{O}_{3}$

\section{PROCEDIMIENTO EXPERIMENTAL}

Con el fin de optimizar el tamaño de partícula y aumentar la reactividad química de los polvos de los materiales de partida durante la sinterización, se ha sometido a los mismos por separado a una molienda de alta energía en un molino de atrición en vía húmeda. El molino de atrición empleado tiene las siguientes características técnicas: capacidad útil de $170 \mathrm{ml}$, velocidad de rotación de $1350 \mathrm{rpm}$ y una potencia de $0.1 \mathrm{Kw}$. El medio de molienda fue alcohol isopropílico y las bolas em- pleadas fueron de Y-PSZ (Tosoh Co.) con $1 \mathrm{~mm}$ de diámetro. La relación en volumen de producto/bolas fue 1:10 y el tiempo de molienda empleado fue de $50 \mathrm{~h}$. Los polvos molidos una vez secados y tamizados se caracterizaron mediante: análisis granulométrico en un analizador de pulso láser de la marca Malvern (modelo Mastersizer), medidas de superficie específica (B.E.T.), difracción de rayos X (DRX) en un Difractómetro Siemens (modelo D 5000), con radiación de $\mathrm{CuK} \alpha(\lambda=1,5406$ $\AA$ ) a una velocidad de barrido de $0.05^{\circ} 2 \theta$ y un voltaje de excitación del ánodo de 50KV, y microscopía electrónica de transmisión (MET) en un microscopio analítico de transmisión (Hitachi, modelo H-7000).

Las imágenes de MET fueron analizadas con un programa de análisis de imagen IMAGIST II SYSTEM, P.G.T. (U.K.), para cuantificar el tamaño y forma de las partículas.

De acuerdo con la sección isopletal $\mathrm{MgO} \bullet \mathrm{CaO}-\mathrm{ZrO}_{2}$ perteneciente al diagrama de equilibrio de fases $\mathrm{ZrO}_{2}-\mathrm{CaO}-\mathrm{MgO}$, calculada anteriormente (28), se seleccionó la composición 84.20 $\mathrm{ZrO}_{2}, 7.90 \mathrm{CaO}$ y $7.90 \mathrm{MgO}$ (\%molar), situada en las cercanías del punto invariante eutectoide de descomposición de la fase cúbica en las fases tetragonal y cúbica de la circona.

La composición seleccionada se sometió a una molienda de alta energía durante $3 \mathrm{~h}$ y los polvos obtenidos, una vez secados y tamizados, fueron prensados isostaticamente a 200Mpa. Se llevaron a cabo tratamientos térmicos a temperaturas de $1000^{\circ}, 1200^{\circ}$ y $1300{ }^{\circ} \mathrm{C}$ con subidas de $20^{\circ} \mathrm{C} / \mathrm{min}$., estabilización durante 18 y 48 minutos y posterior enfriamiento brusco a temperatura ambiente. La evolución de las fases cristalinas y las microestructuras obtenidas en función de la temperatura y tiempo de tratamiento se siguió mediante DRX y microscopía electrónica de barrido (MEB; DSM-950). Para medir la densidad se utilizó el principio de Arquímedes usando mercurio como medio.

\section{RESULTADOS Y DISCUSIÓN}

Considerando polvo nanoparticulado al constituido por partículas de tamaño nanométrico, donde cada una de estas partículas está constituida por "cristalitos" unidos entre sí, formando unidades mayores denominadas "aglomerados" o "agregados" en función del grado de unión entre los cristalitos, el tamaño de partícula observado por ciertas técnicas viene dado por el tamaño de aglomerado o agregado. La aglomeración o agregación puede ser detectada comparando el tamaño de cristalito (medido por DRX o por MET) con el tamaño medio de partícula (medido por técnicas de dispersión de luz-láser y superficie específica).

La circona comercial empleada está en forma de polvo atomizado, y presenta estructura monoclínica (Fig.1a). La superficie específica es $13.5 \mathrm{~m}^{2} / \mathrm{g}$ y el análisis granulométrico revela una distribución multimodal entre 0.1 y $10 \mu \mathrm{m}$ (Fig. 2).

La dolomita $\left(\mathrm{CaMg}\left(\mathrm{CO}_{3}\right)_{2}\right)$ seleccionada fue suministrada en forma de polvo micronizado de elevada pureza. Mediante DRX se observa que no presenta impurezas (Fig. 3a). La superficie específica fue de $2.5 \mathrm{~m}^{2} / \mathrm{g}$ y el análisis granulométrico revela una ancha distribución de tipo bimodal entre 0.2 y 20 $\mu \mathrm{m}$. (Fig. 4).

Los polvos fueron sometidos a distintos tiempos de molienda con el fin de optimizar el proceso de obtención de polvos nanoparticulados. Los resultados del análisis granulométrico mediante pulso láser quedan recogidos en las siguientes 


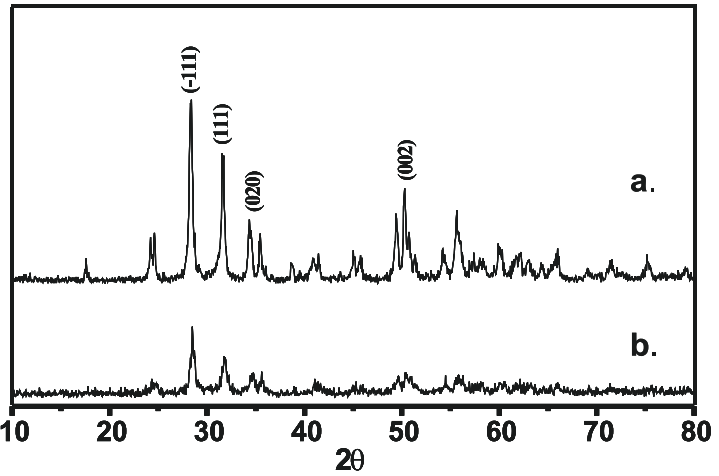

Fig.1 DRX de la $\mathrm{ZrO}_{2}$ de partida (a) y molida 50 horas (b). Se indican las difracciones más características.

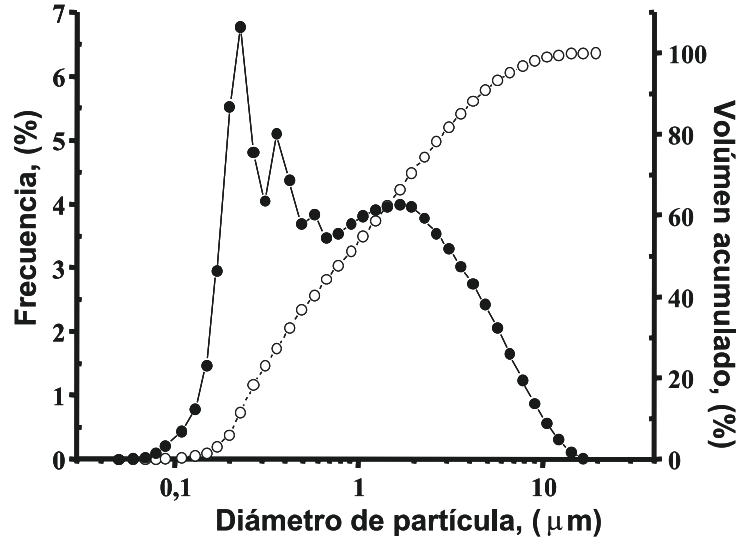

Fig.2 Análisis granulométrico de la $\mathrm{ZrO}_{2}$ de partida.

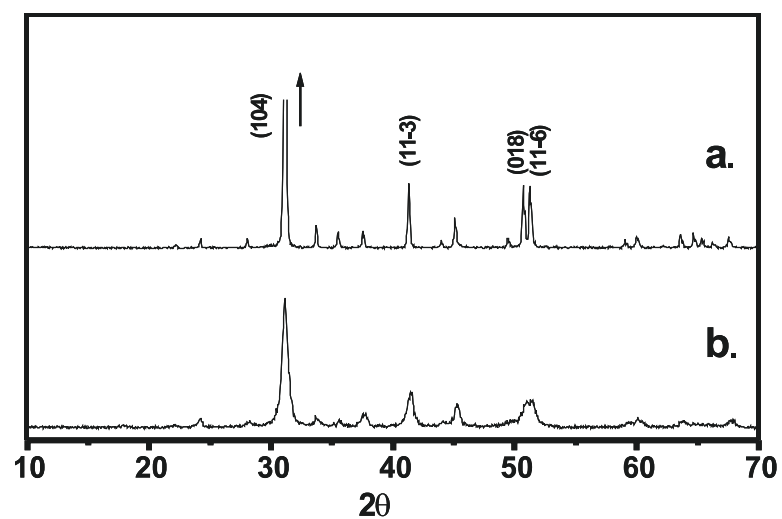

Fig.3 DRX de la $\mathrm{CaMg}\left(\mathrm{CO}_{3}\right)_{2}$ de partida (a) y molida 50 horas (b). Se indican las difracciones más características.

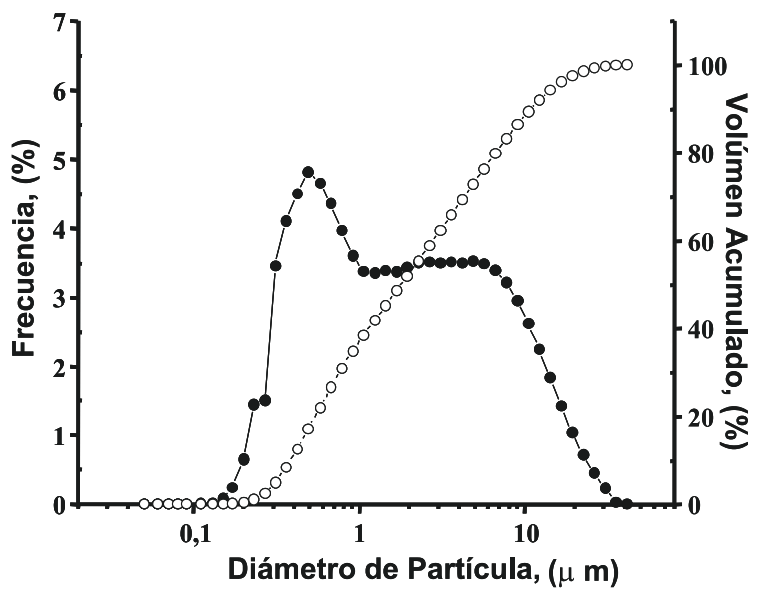

Fig.4 Análisis granulométrico de la $\mathrm{CaMg}\left(\mathrm{CO}_{3}\right)_{2}$ de partida. figuras: en la figura 5 se puede observar el comportamiento de la circona a lo largo de la molienda. En esta figura se observa la disminución progresiva del tamaño de partícula hasta un límite de 33 horas a partir de las cuales se produce un aumento considerable del valor obtenido y esta técnica deja de ser válida para la determinación del tamaño de partícula. En el límite de 33 horas se puede ver un notable estrechamiento de la distribución del tamaño de partícula (Fig. 6) con respecto a la de partida.

La figura 7 recoge igualmente el comportamiento de la dolomita a lo largo de la molienda. En ella puede observarse como se produce la reducción del tamaño de partícula con el tiempo. Para 50 horas de molienda se repite el fenómeno observado en la circona, produciéndose de nuevo un aumento importante del tamaño. Para la dolomita la distribución granulométrica adopta un comportamiento monomodal a tiempos de molienda de 33 horas (Fig.8).

Estos resultados obtenidos por la técnica del analizador de pulso láser, nos indican que para polvos nanoparticulados se produce aglomeración de las partículas durante la medida en el seno de la solución acuosa, debido al aumento en la reactividad de las mismas como consecuencia de la fuerte deformación (disminución del tamaño de partícula y alta superficie específica) y de las imperfecciones estructurales inducidas ambas por la acción mecánica, que hacen que en la superficie se dé una disminución de los números de coordinación atómicos, lo que se traduce en un exceso de polarización superficial que va a activar la superficie aumentando la reactividad entre las partículas(29). Por tanto a la vista de estos resultados se planteó la determinación del tamaño de cristalito mediante Superficie Específica, Difracción de rayos-X y Microscopía Electrónica de Transmisión.

Se midió la superficie específica de los polvos molidos 50 horas obteniéndose valores para la circona de $25 \mathrm{~m}^{2} / \mathrm{g}$ y para la dolomita de $32.5 \mathrm{~m}^{2} / \mathrm{g}$. Comparando estos valores con los de los polvos de partida ( $13.5 \mathrm{~m}^{2} / \mathrm{g}$ y $2.05 \mathrm{~m}^{2} / \mathrm{g}$ respectivamente) se ve un aumento en la superficie específica de los polvos, muy notable en el caso de la dolomita, lo que lleva asociado un incremento substancial en la reactividad de los mismos.

El tamaño de partícula para la circona y para la dolomita puede ser determinado de forma aproximada a partir de estas medidas de superficie específica empleando la siguiente ecuación(30):

$$
D=\frac{6}{\rho \cdot S}
$$

donde $D$ es el diámetro de partícula, $\rho$ la densidad cristalográfica y $S$ la superficie específica. Los resultados obtenidos quedan recogidos en la Tabla I. Resaltar que el valor del diámetro de partícula a partir de medidas de superficie específica corresponde a una superficie que supone el $75 \%$ de la teórica, ya que una fracción importante de área superficial en los cristalitos se pierde debido a los contactos entre los cristalitos y las zonas de los mismos que quedan sin detectar(16) de ahí que los valores reales de tamaño de cristalito se deban ajustar más a los obtenidos por DRX y MET, cuya determinación se detalla a continuación.

Los difractogramas de la $\mathrm{ZrO}_{2}$ y de la $\mathrm{CaMg}\left(\mathrm{CO}_{3}\right)_{2}$ molidas 50 horas (figuras $1 \mathrm{~b}$ y $3 \mathrm{~b}$ ) muestran que la anchura de las bandas ha aumentado debido a la disminución del tamaño de cristalito por efecto de la molienda. Así mismo se observa una disminución en la intensidad de los picos de DRX, debido a la creación de defectos cristalinos y a una pérdida de cristalinidad en los materiales dando lugar a cierta amorfización. 


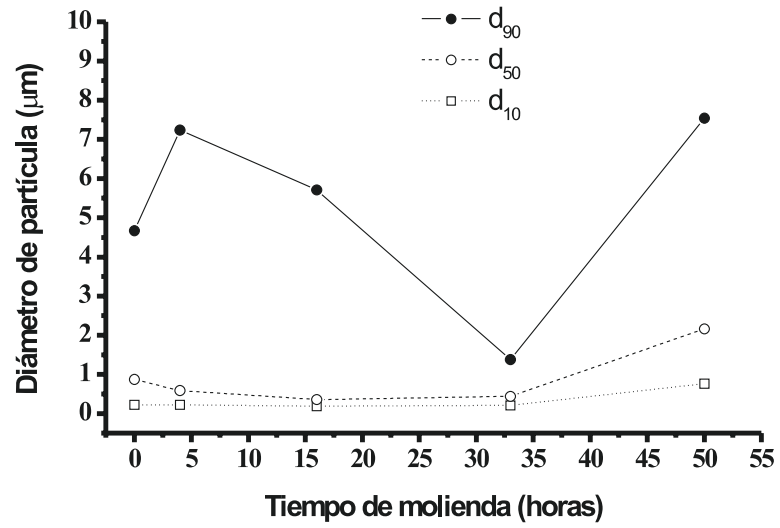

Fig.5 Variación del diámetro de partícula de la $\mathrm{ZrO}_{2}$ con el tiempo de molienda.

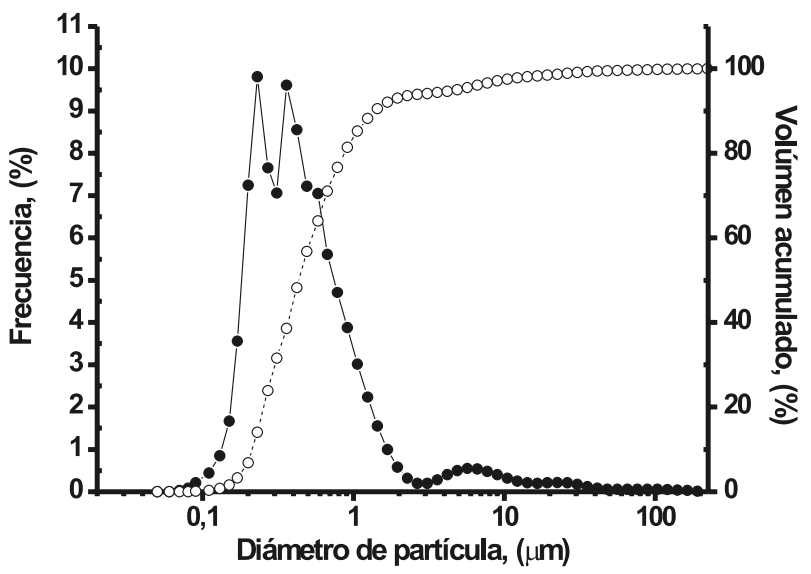

Fig.6 Análisis granulométrico de la $\mathrm{ZrO}_{2}$ molida 33 horas.

Se llevó a cabo un análisis del perfil de las líneas de difracción de rayos $X$ mediante análisis de la relación entre la integral de la anchura y la anchura a media altura de los picos de difracción(31), tomando como funciones de ajuste de la anchura de los picos la suma de las funciones de Gauss y Lorenzt. Las contribuciones debidas a los $\mathrm{K} \alpha_{1}$ y $\mathrm{K} \alpha_{2}$ fueron deconvolucionadas en el programa de evaluación de datos de DRX (Diffract Plus EVA 6.0 Software) que fue usado igualmente para determinar las posiciones de los picos y las fases presentes.

Asumiendo que la anchura de las líneas de DRX para polvos con un tamaño de partícula menor a los $100 \mathrm{~nm}$ es debida a: a) anchura instrumental, b) tamaño de cristalito y c) microtensiones (tensiones a nivel atómico), fue necesario la separación de estos tres componentes para llevar a cabo la determinación del tamaño de cristalito.

La anchura debido al tamaño de cristalito viene dada por la ecuación de Scherrer(31):

$$
\beta_{c}=\frac{K \cdot \lambda}{D_{h k l}} \cdot \cos \theta
$$

donde $\beta_{c}$ es la anchura corregida a la mitad del pico de máxima intensidad, $\mathrm{D}_{\mathrm{hkl}}$ es el tamaño aparente de cristalito en la dirección hkl correspondiente al pico de máxima intensidad, $\mathrm{K}$ es un factor de forma que está comprendido entre 0.9-1 y $\theta$ es el ángulo de Bragg del pico de máxima intensidad. La contribución de las microtensiones a la anchura del pico viene dada por la expresión de Stokes y Wilson:

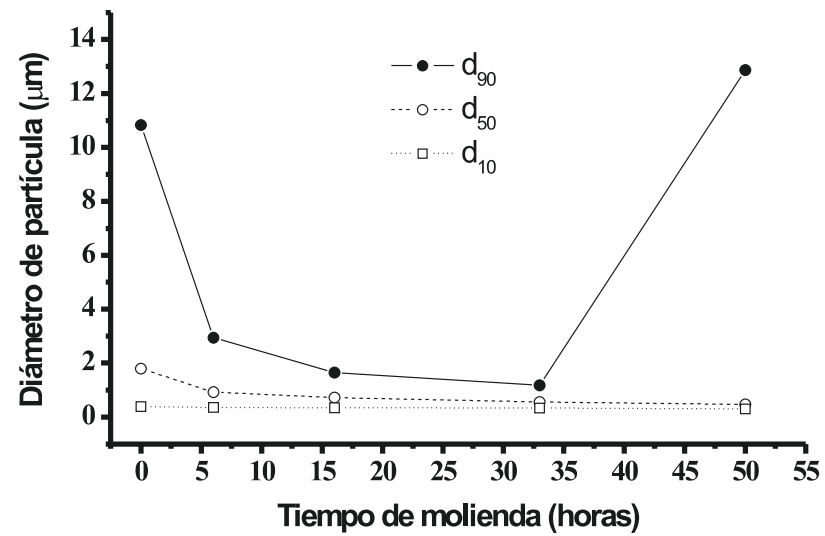

Fig.7 Variación del diámetro de partícula de la $\mathrm{CaMg}\left(\mathrm{CO}_{3}\right)_{2}$ con el tiempo de molienda.

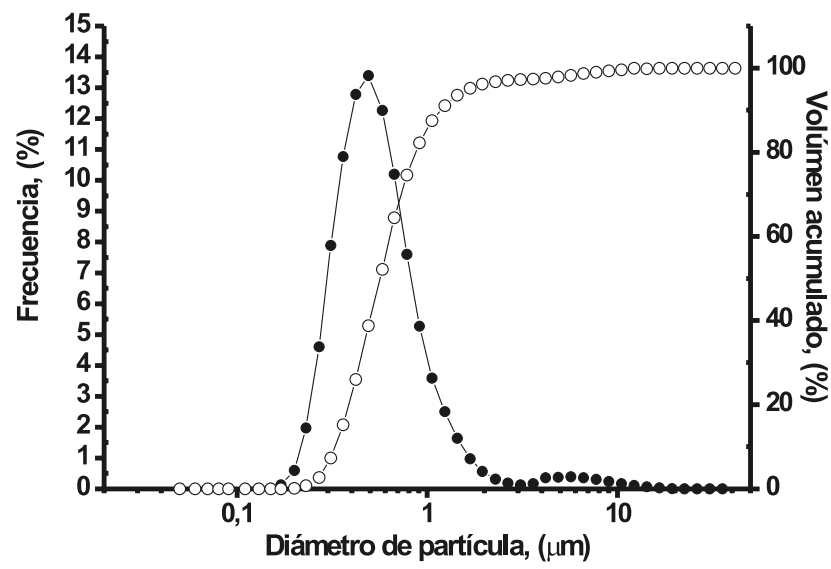

Fig.8 Análisis granulométrico de la $\mathrm{CaMg}\left(\mathrm{CO}_{3}\right)_{2}$ molida 33 horas.

$$
\beta_{t}=\eta_{h k l} \cdot \operatorname{tg} \theta
$$

donde $\eta_{\mathrm{hkl}}$ son las microtensiones en la dirección hkl.

Las microtensiones y el tamaño efectivo fueron estimados por combinación de las ecuaciones anteriores, considerando una distribución de Cauchy para ambas contribuciones, lo que se tradujo en la ecuación de Hall's(32):

$$
\beta_{h k l}=\cos \theta=\frac{K \cdot \lambda}{D_{h k l}^{0}}+\eta_{h k l} \cdot \operatorname{sen} \theta
$$

donde $\beta_{\mathrm{hkl}}$ es la anchura corregida total a la mitad del pico de máxima intensidad y $\mathrm{D}_{\mathrm{hkl}}^{0}$ el tamaño efectivo de cristalito.

\begin{tabular}{|c|c|c|c|c|c|}
\hline \multirow{3}{*}{ 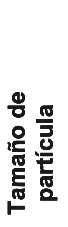 } & & $\begin{array}{c}\mathrm{ZrO}_{2} \\
\text { de partida }\end{array}$ & $\underset{\text { molida } 50 \text { h. }}{\mathrm{ZrO}_{2}}$ & $\underset{\text { de partida }}{\mathrm{CaMg}\left(\mathrm{CO}_{3}\right)_{2}}$ & $\begin{array}{l}\text { CaMg }\left(\mathrm{CO}_{\mathrm{f}}\right)_{2} \\
\text { molida } 50 \mathrm{~h} \text {. }\end{array}$ \\
\hline & $\begin{array}{c}\text { Análisis } \\
\text { pulso láser } \\
\text { (dso) }\end{array}$ & $0.87 \mu \mathrm{m}$ & $0.44 \mu \mathrm{m}$ & $1.79 \mu \mathrm{m}$ & $0.47 \mu \mathrm{m}$ \\
\hline & $\begin{array}{l}\text { Superficie } \\
\text { Específica } \\
\left(\mathrm{m}^{2} / \mathrm{g}\right)\end{array}$ & $76 \mathrm{~nm}$ & $40 \mathrm{~nm}$ & $1 \mu \mathrm{m}$ & $63 \mathrm{~nm}$ \\
\hline \multirow{2}{*}{ 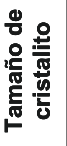 } & M.E.T. & $100 \mathrm{~nm}$ & $20 \mathrm{~nm}$ & $4-5 \mu m$ & $18 \mathrm{~nm}$. \\
\hline & $\begin{array}{l}\text { Difracción de } \\
\text { Rayos X }\end{array}$ & $26 \mathrm{~nm}$ & $24 \mathrm{~nm}$ & $88 \mathrm{~nm}$ & $22 \mathrm{~nm}$ \\
\hline
\end{tabular}

TABLA I.TAMAÑO DE PARTÍCULA/CRISTALITO DETERMINADO A PARTIR DE DIFERENTES TÉCNICAS. 
La relación obtenida entre el tamaño de cristalito efectivo y las microtensiones en los polvos molidos de circona y dolomita de acuerdo a la ecuación \{4\}indicaron que la contribución de las microtensiones a la anchura del pico es prácticamente despreciable. Los tamaños de cristalito aparente fueron determinados aplicando la ecuación $\{2\}$ y quedan recogidos en la Tabla I.

Por MET se ha determinado el tamaño de cristalito de los polvos de los materiales de partida y molidos a distintos tiempos. En la imagen de MET para la circona molida 33 horas (Fig.9) se observan partículas esféricas con un tamaño aproximado de $60 \mathrm{~nm}$. En la imagen de MET para la circona molida 50 horas (Fig.10) se observa un número reducido de partículas con contorno esférico, textura fina y de tamaño aproximado de $60 \mathrm{~nm}$, que recuerdan a las de 33 horas. Estas partículas están rodeadas de aglomerados de un tamaño alrededor de $100 \mathrm{~nm}$, formadas por cristalitos de forma irregular con un tamaño alrededor de 15nm (Fig.11).

En la imagen de MET de la dolomita de partida (Fig.12) se observan partículas de forma alargada con bordes irregulares con un tamaño comprendido en un intervalo de 2-6 $\mu \mathrm{m}$. En la imagen de MET de la dolomita molida 50 horas (Fig.13) se observan partículas con un tamaño alrededor de $80 \mathrm{~nm}$ con forma irregular y de contorno angular que están agrupados formando aglomerados de mayor tamaño. A mayores aumentos (Fig.14) es posible observar que estos aglomerados están constituidos por cristalitos con un tamaño alrededor de $18 \mathrm{~nm}$. La creación de partículas con forma de placas irregulares y la presencia de defectos en las mismas, es debido a la acción de las fuerzas de cizalla generadas por el proceso de atrición mecánica durante la molienda de alta energía.

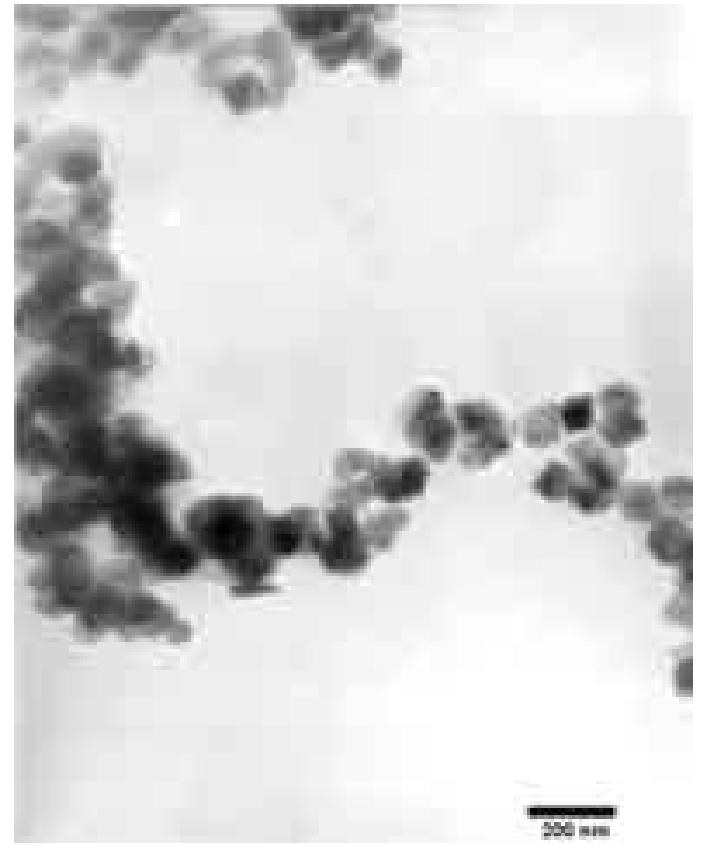

Fig. 9 Imágen MET de la $\mathrm{ZrO}_{2}$ molida 33 horas.

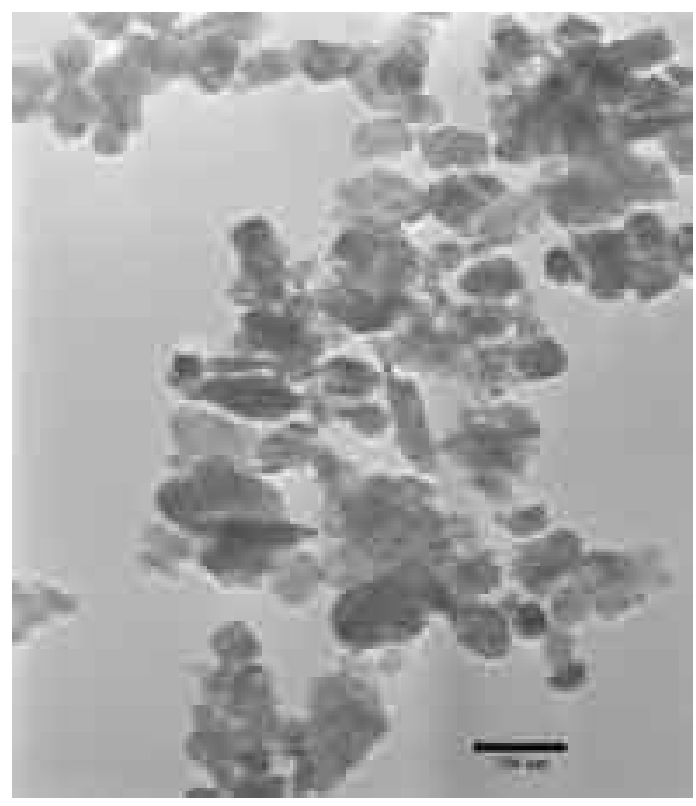

Fig. 10 Imágen MET de la $\mathrm{ZrO}_{2}$ molida 50 horas.

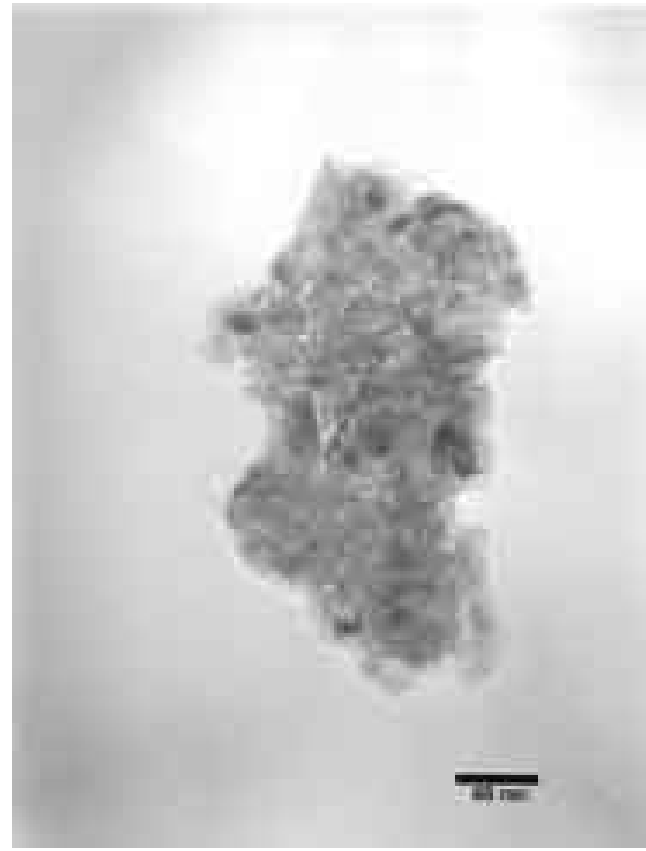

Fig. 11 Imágen MET: aglomerado de cristalitos de $\mathrm{ZrO}_{2}$ molida 50 horas.

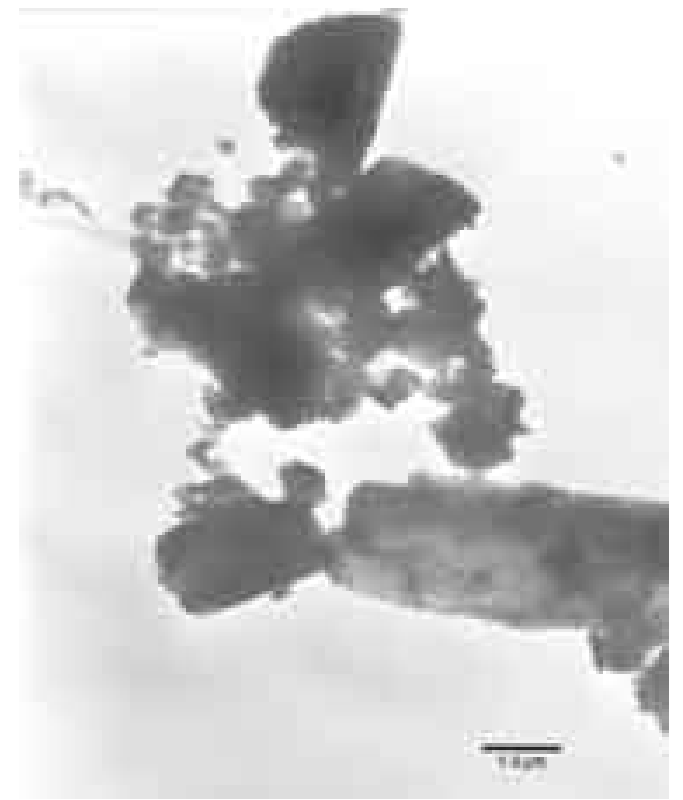

Fig.12 Imágen MET de la Dolomita de partida. 


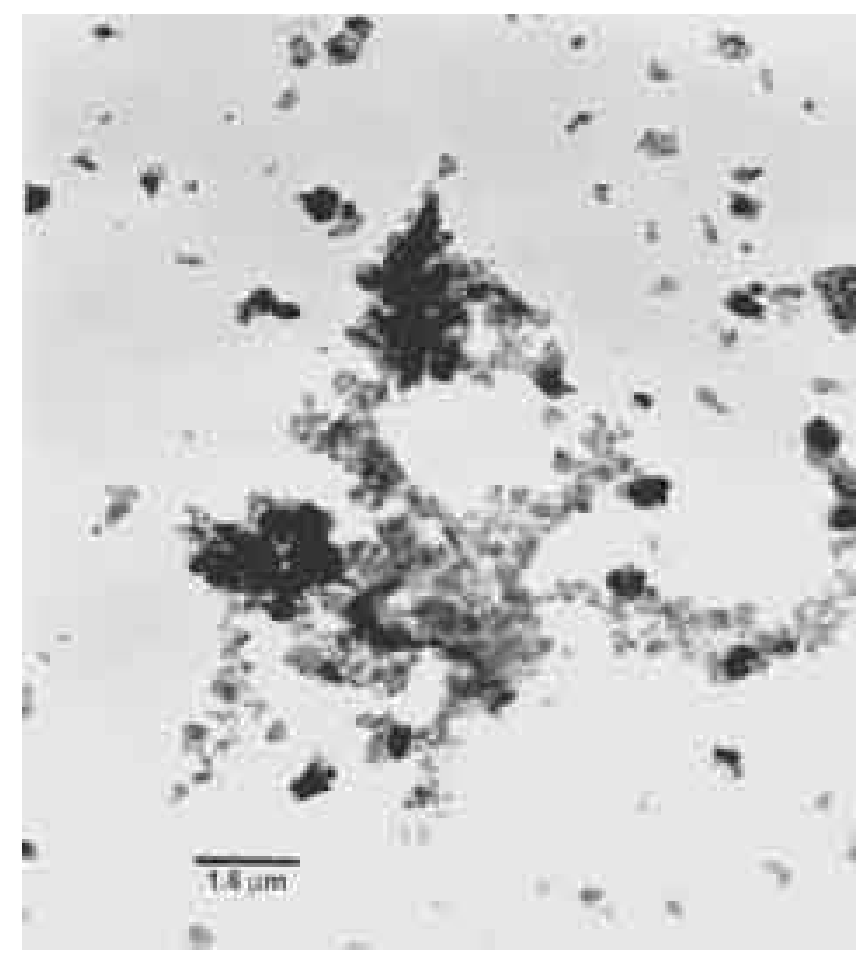

Fig. 13 Imágen MET de Dolomita molida 50 horas.

Comparando la dolomita molida 50 horas con la dolomita de partida es visible como las partículas iniciales, bloques alargados con forma irregular de 2-6 $\mu \mathrm{m}$, se han fracturado por efecto de la molienda dando lugar a cristalitos con un tamaño alrededor de $18 \mathrm{~nm}$. Estos resultados muestran que como era de esperar la atrición mecánica es más efectiva para el caso de la dolomita (que posee grupos carbonato) dando lugar a polvos con una alta superficie específica, bajo tamaño de partícula y una alta amorfización del material como se demostró anteriormente por DRX.

El presente estudio ha permitido determinar las características óptimas para obtener materiales de circona (Ca, $\mathrm{Mg}$ PSZ) nanocristalina mediante mezcla de polvos, previamente molidos, de circona monoclínica y dolomita con estequiometría adecuada (84.20 $\mathrm{ZrO}_{2} 7.90 \mathrm{CaO}$ y $7.90 \mathrm{MgO}$ \% \%olar) y seleccionada a partir de la región isopletal $\mathrm{MgO} \cdot \mathrm{CaO}-\mathrm{ZrO}_{2}$ propuesta anteriormente por Serena y col.(28).

Tratamientos térmicos a baja temperatura $\left(1000^{\circ} \mathrm{C} / 18 \mathrm{~min}\right.$. $)$ dan lugar a materiales con una densidad de $\approx 90 \%$ respecto a la teórica y una microestructura formada por granos con un tamaño aproximado de $100 \mathrm{~nm}$ (Fig.15). Las fases observadas por DRX son monoclínica, cúbica y tetragonal. La existencia de circona monoclínica informa de que la temperatura y tiempo empleados no han sido suficientes para que la transformación sea completa.

Tratamientos a $1200^{\circ} \mathrm{C} / 48 \mathrm{~min}$ y $1300^{\circ} \mathrm{C} / 18 \mathrm{~min}$ dieron lugar a materiales de circona (Ca, Mg-PSZ) bien sinterizados con densidades próximas a las teóricas (96-98\%), con un crecimiento pequeño de grano, aspecto remarcable si se compara con los materiales de PSZ que generalmente se obtienen por otros procesos(33). El estudio por DRX de estas muestras (Fig.16) confirma que el material está compuesto por las fases cúbica y tetragonal. La existencia de circona tetragonal en la matriz de circona cúbica se pudo comprobar llevando a cabo un di-

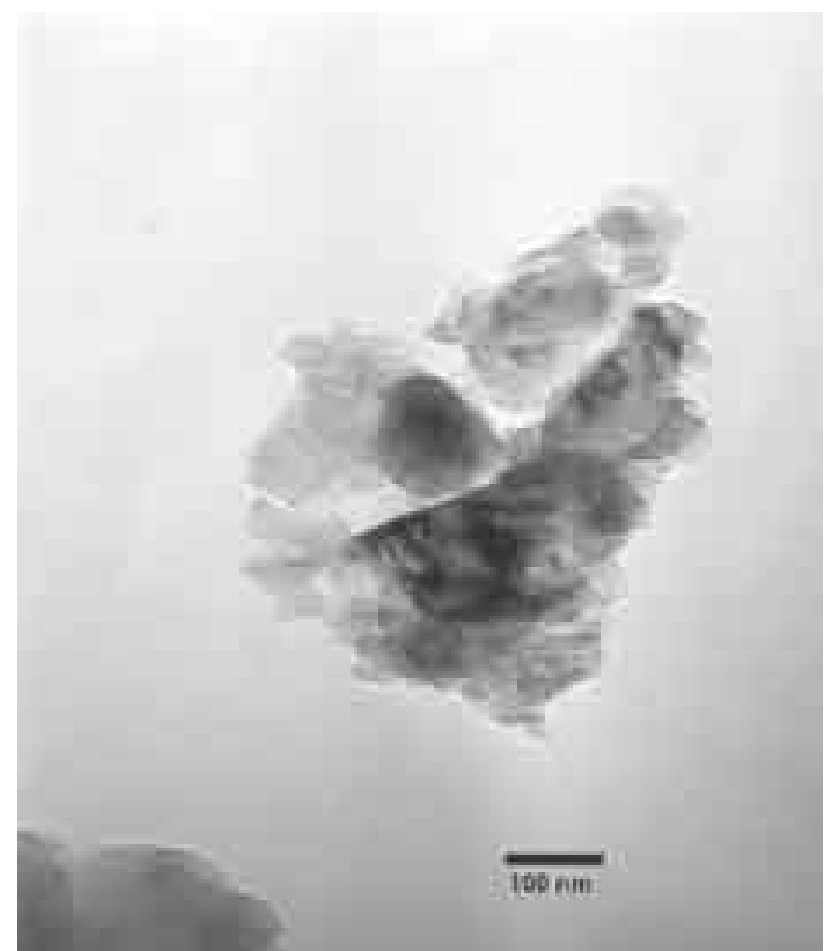

Fig. 14 Imágen MET: aglomerado de cristalitos de dolomita molida 50 horas.

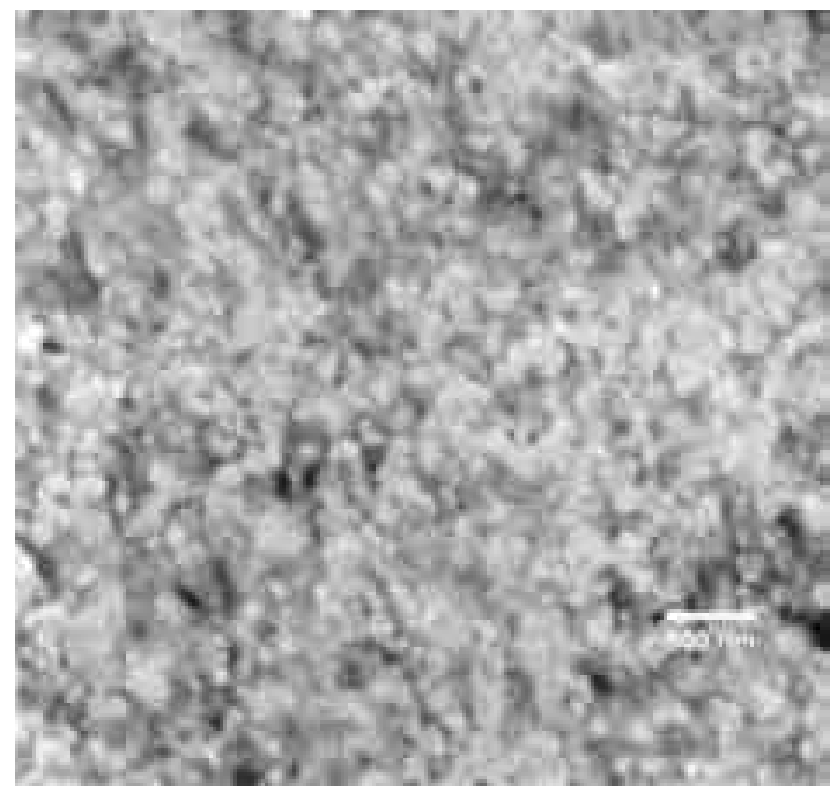

Fig. 15 Imágen MEB de la muestra tratada a $1000^{\circ} \mathrm{C} / 18$ minutos.

fractrograma de rayos- $X$ a una velocidad de barrido de $0.05^{\circ}$ $2 \theta$ y tiempos de 10 segundos en el intervalo $73-75^{\circ}(2 \theta)$. En la figura 16 se muestra la existencia de la familia de planos $\{400\}$ de la fase cúbica, $\{004\}$ y $\{220\}$ de la fase tetragonal junto con las deconvoluciones de los mismos. En la caracterización microestructural de estas muestras (Fig.17) se observan precipitados esféricos muy pequeños $(\sim 30-40 \mathrm{~nm})$ de circona tetragonal, similares a los producidos durante el enfriamiento para ciertas composiciones en los sistemas $\mathrm{Ca}-\mathrm{ZrO} 2$ y $\mathrm{MgO}-\mathrm{ZrO}_{2}(34,35)$. 
Tratamientos a tiempos mayores $\left(1300^{\circ} \mathrm{C} / 48 \mathrm{~min}\right)$ dieron como resultado el crecimiento de la fase tetragonal a partir de los precipitados esféricos obteniéndose el engrosamiento de ciertos de ellos (Fig.18). Tratamientos posteriores a temperaturas superiores permitieron establecer la existencia de la fase cúbica como única fase estable, concordando esto último con la sección isopletal anteriormente calculada (28).

En general los materiales de PSZ que aparecen en la literatura (24,33-37) con densidades semejantes a las obtenidas en el presente trabajo han sido tratados en el intervalo de temperaturas de $1700^{\circ} \mathrm{C}-2100^{\circ} \mathrm{C}$ presentando tamaños de grano para la fase cúbica y tetragonal de la circona mucho más elevados (50-200 $\mu \mathrm{m}$ y $\sim 1-2 \mu \mathrm{m}$ respectivamente) y necesitando largos periodos de envejecimiento para poder cristalizar la fase tetragonal.

Los resultados obtenidos en la optimización del tamaño de partícula muestran que la molienda de alta energía es efectiva para la obtención de polvo nanoparticulado tanto de circona monoclínica como de dolomita. El efecto obtenido es un incremento substancial de la energía libre superficial de las partículas y una disminución de los caminos de difusión, lo que provoca un aumento en la reactividad de los polvos resultantes pudiéndose obtener materiales de $\mathrm{Ca}, \mathrm{Mg}$-PSZ bien sinterizados a baja temperatura $\left(1200^{\circ} \mathrm{C}\right)$.

\section{CONCLUSIONES}

- La activación mecánica ha resultado ser más efectiva para la dolomita que para la circona. Este proceso permitió obtener cristalitos entre 15 y $20 \mathrm{~nm}$ y aglomerados de dichos cristalitos con un tamaño medio menor de $100 \mathrm{~nm}$ para ambos materiales.

- Se han obtenido circonas parcialmente estabilizadas (Ca,Mg-PSZ) nanocristalinas con densidades del $98 \%$ respecto a la teórica, a temperaturas tan bajas como $1200^{\circ} \mathrm{C}$. La composición elegida da lugar a una solución sólida de $\mathrm{CaO}$ y $\mathrm{MgO}$ en la $\mathrm{ZrO}_{2}$ a las temperaturas de trabajo y proporciona la formación de precipitados nanocristalinos de fase tetragonal en una matriz de circona cúbica.

- La microestructura de estos materiales consiste en una matriz cúbica de granos de circona con un tamaño entre 2-5 $\mu \mathrm{m}$ conteniendo precipitados de circona tetragonal con un tamaño medio aproximado de $30 \mathrm{~nm}$ para las temperaturas mas bajas y hasta $200 \mathrm{~nm}$ en el caso de las muestras a $1300^{\circ} \mathrm{C} / 48 \mathrm{~min}$.

\section{AGRADECIMIENTOS}

El trabajo ha sido realizado gracias a la financiación de los proyectos CAM 07N/0038/2001 y CICYT MAT 2001-941.

\section{BIBLIOGRAFÍA}

1. M.J. Mayo: "Processing of Nanocrystalline Ceramics From Ultrafine Particles", International Materials Reviews Vol. 41 No.3., 1996.

2. H. Gleiter: "Nanocrystalline Materials", Progress in Materials Science Vol, 33, pp. 223-315, 1989

3. R. W. Siegel: "Nanostructured Materials: Mind Over Matter", Nanostructured Materials, Vol. 3, pp. 1-19, 1993

4. K. Niihara: "New Desingn Concept of Structural Ceramics: Ceramics Nanocomposites", Journal of The Ceramic Society of Japan, 99 [10] 1991.

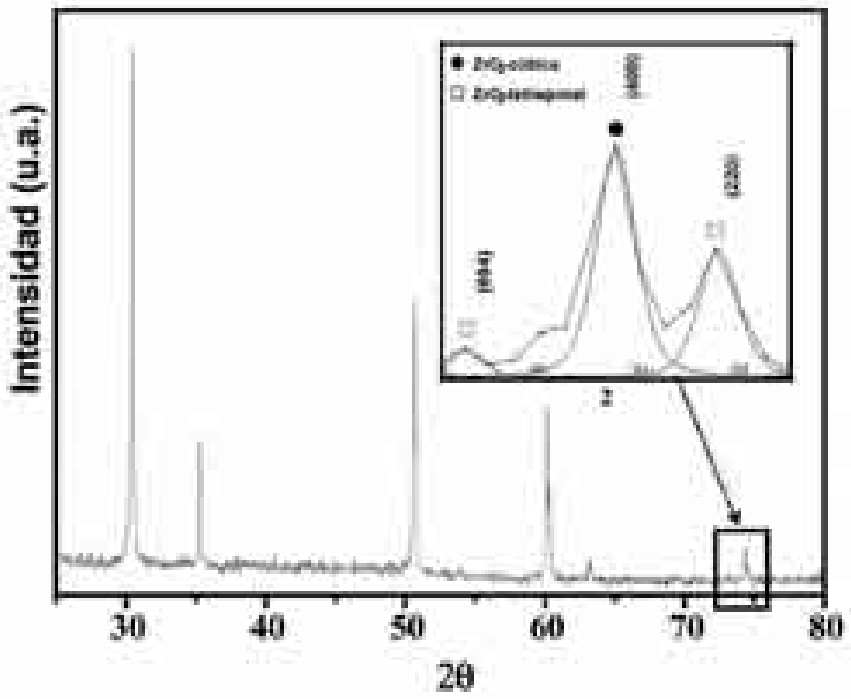

Fig. 16 DRX de la muestra tratada a $1200^{\circ} \mathrm{C} / 48$ minutos. La zona ampliada muestra la deconvolución de los picos de DRX pertenecientes a la familia de planos $\{400\}$ de las fases cúbica y tetragonal de la circona en el intervalo $73-75^{\circ} 2 \theta$.

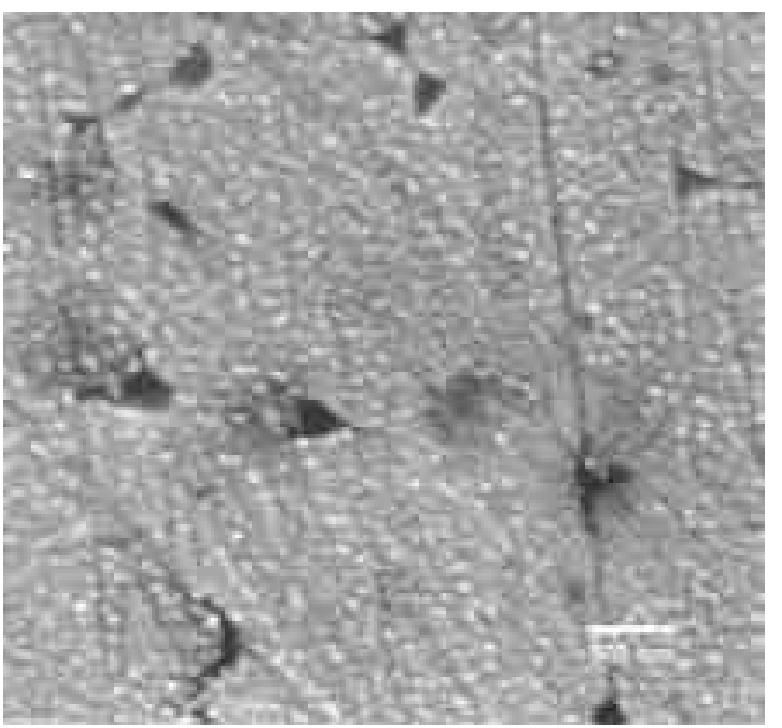

Fig. 17. Imágen de $\mathrm{MEB}$ de la muestra tratada a $1300^{\circ} \mathrm{C} / 18$ minutos.

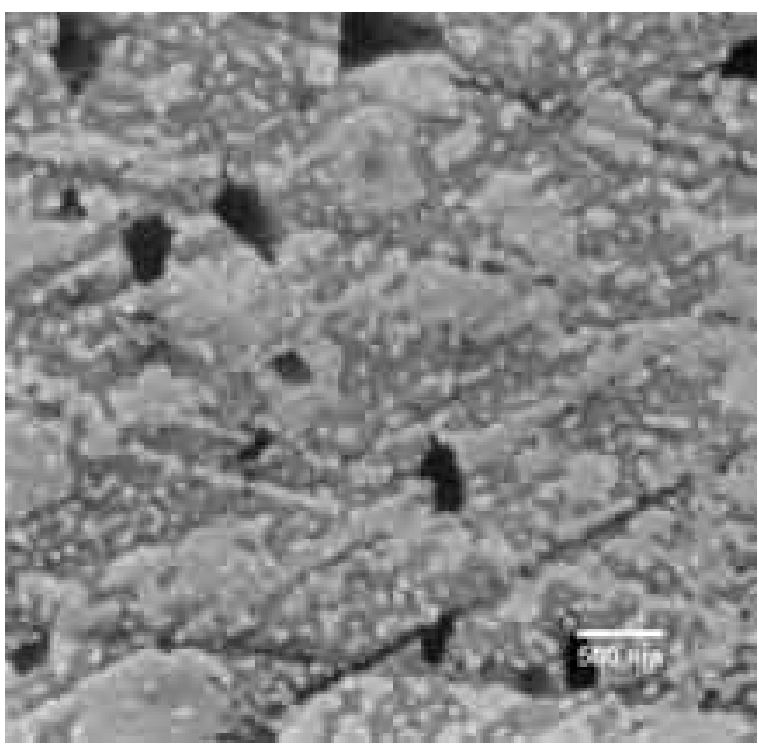

Fig. 18. Imágen de $\mathrm{MEB}$ de la muestra tratada a $1300^{\circ} \mathrm{C} / 48$ minutos. 
5. C.C. Koch: "The Synthesis and Structure of Nanocrystalline Materials Produced by Mechanical Attrition: a Review", Nanostructured Materials Vol. 2, pp. 109-129, 1993.

6. F.H. (Sam) Froes et al.: "Synthesis of Nanocrystalline Materials - an Overview", Materials Science and Engineering A301 (2001) 44-53.

7. H.J. Fecht: "Nanostructure Formation by Mechanical Attrition", Nanostructured Materials, Vol. 6, pp. 33-42, 1995.

8. C. Suryanarayana: "Mechanical Alloying and Milling", Progress in Materials Science 46 (2001) 1-184.

9. Z. Brankovic et al.: "Mechanochemical Synthesis of PZT Powders", Materials Science and Engineering, 345 (2003) 243-48.

10. Kenneth J.D. MacKenzie et al.: "Mechanochemical Processing of Sialon Compositions", Journal of the European Ceramic Society 23 (2002).

11. Kalpana Awasthi et al.: "Ball-Milled Carbon and Hydrogen Storage", International Journal of Hydrogen Energy 27 (2002) 425-432.

12. J.M. Xue et al.: "Funcional Ceramics of Nanocrystallinity by Mechanical Activation", Solid State Ionics 151 (2002) 403-412.

13. Jadambaa Temuujin et al.: "Effect of Mechanochemical Treatment on the Synthesis of Calcium Dialuminate", J. Mater Chem. 2000. 10. 1019-1023.

14. P.N. Kuznetsov et al.: “Ultra Fast Synthesis of Metastable Tetragonal Zirconia by Means of Mechanochemical Activation", Applied Catalysis A: General 227 (2002) 299-307.

15. L.B. Kong et al.: " $\mathrm{MgAl} \mathrm{O}_{2}$ Spinel Phase Derived From Oxide Mixture Activated by a High-Energy Ball Milling Process", Materials Letters 56 (2002) 238-243.

16. G. Skandan: "Processing of Nanostructured Zirconia Ceramics", Nanostructured Materials, Vol. 5, No. 2, pp. 111-126, 1995.

17. S.Somiya, N. Yamamoto and H. Yanagida, Eds., "Science and Technology of Zirconia III," Advances in Ceramics, Vols. 24A and 24B. American Ceramic Society, Westerville, $\mathrm{OH}, 1986$

18. S.Appel, R. Clasen, A. Chkourankov, H. Natter, R. Hempelmann, S. Schlabach, B. $\mathrm{Xu}$ and D. Vollath: "Comparative Investigation of $\mathrm{Al}_{2} \mathrm{O}_{3}$ - and $\mathrm{ZrO}_{2}$ Nanopowders Synthesized by Different Methods", p.p. 585-600 in Ceramic Engineering and Science Proceedings vol. 23, Issue 4, " $26^{\text {th }}$ Annual Conference on Composites, Advanced Ceramics, Materials, and Structures: B", Cocoa Beach, Florida, January 13-18,2002. Ed. H.T. Lin and M. Singh, The American Ceramic Society.

19. S.K. Tadokoro, E.N.S. Muccillo: "Synthesis and Characterization of Nanosized Powders of Yttria-Doped Zirconia", J. of Alloys and Compounds 344 (2002) 186-189.

20. D. G. Lamas et al: "Synthesis and Characterization of Nanocrystalline Powders for Partially Stabilized Zirconia Ceramics", J. European Ceramic Soc. 18 (1998) 1217-1221.

21. U. Betz et al.: "Microstructural Development During Final-Stage Sintering of Nanostructured Zirconia Based Ceramics", Materials Science and Engineering A281 (2000) 68-74.
22. S.D. Ramanurthi, et al: "Nanometer-Sized $\mathrm{ZrO}_{2}$ Particles Prepared by a SolEmulsion-Gel Method", J. Am. Ceram. Soc. 73 [9] 2760-63 (1990).

23. Pol Duwez et al: "Stabillization of Zirconia with Calcia and Magnesia", J. Am. Ceram. Society, May 1, 1952.

24. R.M. Dickerson and A.H. Heuer: "Precipitate Morphology in Ternary MgO, CaO-Partially-Stabilized Zirconias", J. Am. Ceram. Soc. 76 [4] 833-40 (1993).

25. V.S. Stubican and J.R. Hellmann: "Phase Equilibria in Some Zirconia Systems", pp.25-36 IN Advanced Ceramics, Vol 3, Science and Technology of Zirconia. Edited by A.H. Heuer and L.W. Hobbs. American Ceramic Society, Columbus, $\mathrm{OH}, 1981$

26. Joanna R.Groza: “Nanosintering”, Nanostructured Materials, Vol 12, pp.987992, 1999.

27. A. Kats-Demyanets et al.: "Grain Growth Control in Nanocrystalline Oxide Ceramics", Nanostructured Materials, Vol 6, pp. 851-854, 1995.

28. S. Serena, M.A. Sainz, S. de Aza, A. Caballero, "Thermodynamic assessment of the System $\mathrm{ZrO}_{2}-\mathrm{CaO}-\mathrm{MgO}$ using new experimental results. Calculation of the isoplethal section $\mathrm{MgO} \cdot \mathrm{CaO}-\mathrm{ZrO}_{2}$ " enviado al J.Am.Ceram. Soc.

29. J.C. Ray et al: "Formation of $\mathrm{Cr}^{3+}$ Stabilized $\mathrm{ZrO}_{2}$ Nanocrystals in a Single Cubic Metastable Phase by a Novel Chemical Route with a SucrosePolyvinyl Alcohol Polymer Matrix", Materials Letters 48 (2001) 281-291.

30. R.C. Garvie: "The Ocurrence of Metastable Tetragonal Zirconia as a Crystallite Size Effect", The Journal of Physical Chemistry, Vol. 69, No. 4, April 1965.

31. Klug H.P. and Alexander I.E.: "X-Ray Diffraction Procedure for Polycrystalline and Amorphous Materials". Ed. John Wiley and Sons, New York, 1974.

32. B. Lönnberg: "Characterization of Milled $\mathrm{Si}_{3} \mathrm{~N}_{4}$ Powder Using X-Ray Peak Broadening and Surface Area Analysis", Journal of Materials Science 29 (1994) 3224-3230.

33. J.F. Jue: "Fabrication, Microstructural Characterization, and Mechanical Properties of Polycrystalline T'-Zirconia", J. Am. Ceram. Soc. 73 [12] 365057 (1990).

34. R.R.Hugan and R.H.J.Hannink: "Precipitation During Controlled Cooling of Magnesia-Partially-Stabilized Zirconia", J. Am. Ceram. Soc. 69 [7] 556-63 (1986)

35. V.Lanteri et al.: “Morphology of Tetragonal Precipitates in Partially Stabilized $\mathrm{ZrO}_{2}{ }^{\prime}$, J. Am. Ceram. Soc.69 [7] 564-69 (1986).

36. E.P. Butler and A.H. Heuer: "Grain-Boundary Phase transformations During Aging of a Partially Stabilized $\mathrm{ZrO}_{2}$ - A liquid-Phase Analogue of DiffusionInduced Grain-Boundary Migration (DIGM) (?)", J. Am. Ceram. Soc., 68 [4] 197-202 (1985).

37. J.M. Marder et al: "Precipitation From Cubic $\mathrm{ZrO}_{2}$ Solid Solutions", Acta metall. Vol.31, No.3, pp.387. 1983

Recibido: 04.06.03

Aceptado: 07.07.03 